\title{
Preliminary Biometrics of ECG Signal Based on Temporal Organization through the Implementation of a Multilayer Perceptron Neural Network
}

\author{
Robert LeMoyne', Timothy Mastroianni² \\ ${ }^{1}$ Department of Biological Sciences, Northern Arizona University, Flagstaff, USA; ${ }^{2}$ Cognition Engineering, \\ Pittsburgh, USA
}

Correspondence to: Robert LeMoyne, rlemoyne07@gmail.com

Keywords: ECG Signal, Biometrics, Multilayer Perceptron Neural Network, Machine Learning, Signal Analysis Received: November 1, $2021 \quad$ Accepted: December 20, $2021 \quad$ Published: December 23, 2021

Copyright $\odot 2021$ by author(s) and Scientific Research Publishing Inc.

This work is licensed under the Creative Commons Attribution International License (CC BY 4.0).

http://creativecommons.org/licenses/by/4.0/

\section{(c) (1) Open Access}

\section{ABSTRACT}

The attributes of the ECG signal signifying the unique electrical properties of the heart offer the opportunity to expand the realm of biometrics, which pertains the identification of an individual based on physical characteristics. The temporal organization of the ECG signal offers a basis for composing a machine learning feature set. The four attributes of the feature set are derived through software automation enabled by Python. These four attributes are the temporal differential of the $P$ wave maximum and $T$ wave maximum relative to the $R$ wave maximum and the $Q$ wave minimum and $S$ wave minimum relative to the $R$ wave maximum. The multilayer perceptron neural network was applied and evaluated in terms of classification accuracy and time to develop the model. Superior performance was achieved with respect to a reduced feature set considering only the temporal differential of the $P$ wave maximum and $T$ wave maximum relative to the $R$ wave maximum by comparison to all four attributes applied to the feature set and the temporal differential of the $Q$ wave minimum and $S$ wave minimum relative to the $R$ wave maximum. With these preliminary findings and the advent of portable and wearable devices for the acquisition of the ECG signal, the temporal organization of the ECG signal offers robust potential for the field of biometrics.

\section{INTRODUCTION}

The domain of biometrics is a rapidly advancing field that provides verification of an individual based on the individual's uniquely intrinsic physiological characteristics [1-3]. For example, a standard biometric application pertains to a person's fingerprint. More sophisticated biometric techniques involve identification of the voice, face, and aspects of the eye, such as the retina and iris [1]. 
Intuitively, functionality of the human heart is a candidate for the realm of biometrics. The electrocardiogram (ECG) signal ascertains the electric properties of the heart, which also infers the mechanical functionalities $[4,5]$. Recently, the feasibility of ECG biometrics has rapidly evolved especially with the development of relevant wearable applications $[2,3,6]$.

The machine learning algorithm known as the multilayer perceptron neural network provides an intuitive means for attaining a classification accuracy that is representative of the neurological foundation for the brain [7-12]. Additionally, the application of clinically perceptible attributes, such as utilizing the temporal domain of the ECG signal, to define the machine learning feature set may enhance comprehension among experts that are more peripheral to the domain of machine learning. Therefore, the objective of the research endeavor was to apply the multilayer perceptron neural network machine learning algorithm to distinguish disparate ECG signals of subjects based on temporal organization of the ECG signals with a dual emphasis respective of classification accuracy and time to develop the model.

\section{BACKGROUND}

\subsection{A Perspective of Biometrics}

Biometrics has been identified as an emerging technology with considerable impact. This technology pertains to the identification of people based on their inherently specific physiological attributes. The field of biometrics is highly relevant to personal security, such as the authentication of a person's identity [1]. Central to the feasibility of a biometric application is the capacity to acquire the relevant signal data in a convenient means, such as enabled through portable and wearable devices for obtaining ECG signal data $[1-3,6]$. The ECG signal is particularly of interest as a candidate for biometric applications, since the ECG signal is highly robust to counterfeiting and feasible for continuous measurement $[2,3,13]$.

\subsection{A Perspective of the Electrocardiogram (ECG) and $P, Q, R, S$, and T Waves of the ECG Signal}

The origins of the development of the first clinical electrocardiograph for ascertaining the electrocardiogram (ECG) are attributed to Willem Einthoven, for which he was awarded the Nobel Prize during 1924 [5]. The opportunity for applying the electrocardiograph as a clinical diagnostic system and now for biometric applications has been considerably augmented with progressive evolution from the original device requiring two rooms with a weight of approximately 600 pounds to current wearable and inherently portable applications $[5,6]$. The ECG signal reveals considerable diagnostic insight with respect to the health status of the heart $[5,14,15]$. The ECG signal reveals highly perceptible features, such as the $\mathrm{P}, \mathrm{Q}$, $\mathrm{R}, \mathrm{S}$, and $\mathrm{T}$ waves $[4,5]$.

The P, Q, R, S, and T waves of the ECG signal constitute distinct aspects of the activity of the heart [4, 5]. The $P$ wave can be segmented into two aspects in order to determine activity of the two atriums of the heart. The first half of the $\mathrm{P}$ wave pertains to the depolarization of the right atrium, and the second half of the $\mathrm{P}$ wave is representative of the depolarization of the left atrium [4].

Subsequent to the P wave is the QRS complex, which derives multiple aspects of the heart's functionality. Preliminarily, the middle section of the left aspect for the interventricular septum undergoes depolarization. Then both ventricles' endocardium undergoes depolarization, which is followed by decreasing right ventricle depolarization and increasing left ventricle depolarization. The terminal portion of the QRS complex represents the basilar aspect of the left ventricle undergoing depolarization [4].

After the QRS complex is completed the $\mathrm{T}$ wave commences. The $\mathrm{T}$ wave is representative of the repolarization of the ventricles [4]. A representative ECG signal is presented in Figure 1. Note that the P, R, and $\mathrm{T}$ waves involve maxima. The $\mathrm{R}$ wave constitutes a global maximum, and the $\mathrm{P}$ and $\mathrm{T}$ waves feature local maxima. The $\mathrm{Q}$ and $\mathrm{S}$ waves additional present minima. Additionally, the $\mathrm{R}$ wave with its global maximum presents the most readily identifiable feature.

From a high level perspective machine learning involves consolidating signal data to a numerical feature set consisting of numeric attributes [16]. For example, with respect to neurologically and biomechanically 


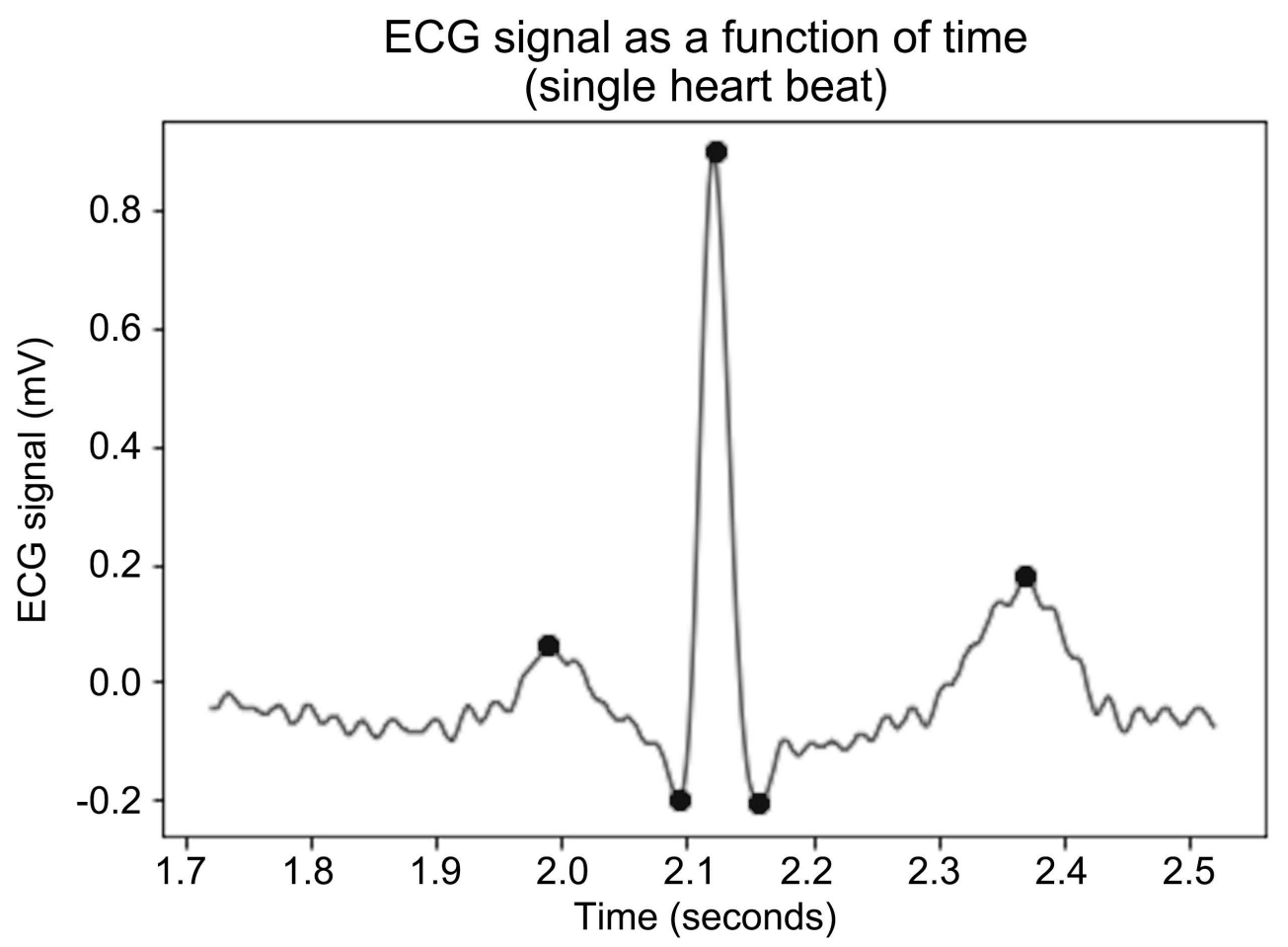

Figure 1. An ECG signal illustrating the $P, Q, R, S$, and $T$ waves with their representative associated maxima and minima, which are emphasized by a filled dot. The $P, Q, R, S$, and $T$ waves proceed from left to right.

themed signal data, the temporal organization of maxima and minima provides an instrumental basis for developing a feature set that achieves considerable classification accuracy [17, 18]. Based on the visualization of a representative ECG signal from Figure 1, the temporal organization of the $\mathrm{R}$ wave relative to the $\mathrm{P}, \mathrm{Q}, \mathrm{S}$, and $\mathrm{T}$ waves derives a feature set with the opportunity for achieving considerable classification accuracy. The selection of an appropriate machine learning classification algorithm that provides a visualized presentation for the basis of achieving distinctive classification accuracy that would be comprehensible to experts contributing to biometrics that are extrinsic to the domain of machine learning would be beneficial.

\subsection{The Fundamentals of the Multilayer Perceptron Neural Network}

An advantage of the multilayer perceptron neural network is its intuitive semblance from a computational perspective to the neuron, which is a foundational for the perceptive capabilities of the brain [7-12]. Inherent features of the multilayer perceptron neural network involve the use of backpropagation and the sigmoid function. Additionally, the multilayer perceptron neural network has been applied to numerous applications for distinguishing human health status based on signal data [7, 19]. The research endeavor objective is to utilize the multilayer perceptron neural network to distinguish subjects based on the temporal organization of their available ECG signal data with consideration of both classification accuracy and time to develop the model.

\section{MATERIALS AND METHODS}

The ECG signal data was acquired through the online resource PhysioNet. PhysioNet provides global access to an assortment of clinical and physiological signal data, such as the ECG signal, the platform is maintained by MIT Laboratory for Computational Physiology [20, 21]. The research was conducted from 
a perspective of engineering proof of concept. ECG signal data was selected from two subjects with a sampling rate of $500 \mathrm{~Hz}$ and downloaded in CSV format for post-processing by Python.

Python was applied to consolidate the ECG signal data to a feature set to an Attribute-Relation File Format (ARFF) suitable for the Waikato Environment for Knowledge Analysis (WEKA) [10-12]. The following procedure generalizes the Python software program from a pseudo code perspective:

1) Upload the ECG signal data for Python post-processing.

2) Acquire the temporal location of the $R$ wave maximum for the first 10 heart beats.

3) Based on temporal location of the $R$ wave maximum acquire the maximum of the $P$ wave and maximum of the $\mathrm{T}$ wave.

4) Acquire the temporal location of the minima for the $Q$ wave and $S$ wave based on the temporal location of the maxima for the $\mathrm{R}$ wave, $\mathrm{P}$ wave, and $\mathrm{T}$ wave.

5) Visualize the maxima of the $P$ wave, $R$ wave, and $T$ wave and the minima of the $Q$ wave and $S$ wave in as illustrated in Figure 1.

6) Determine the temporal differential of the maximum of the $P$ wave and the maximum of the $T$ wave with respect to their associated $\mathrm{R}$ wave maximum.

7) Determine the temporal differential of the minimum of the $Q$ wave and the minimum of the $S$ wave with respect to their associated $\mathrm{R}$ wave maximum.

8) Write the temporal differentials for the $P, Q, S$, and $T$ waves with respect to their associated $R$ wave to an ARFF file for machine learning classification.

9) Apply the above process for both ECG signals of the two subjects.

A multilayer perceptron neural network available through WEKA has been selected as the algorithm to conduct machine learning classification. Tenfold cross-validation was applied [10-12]. The generated multilayer perceptron neural network was subsequently evaluated in terms of classification accuracy and time to build the model.

\section{RESULTS AND DISCUSSION}

The Python software enabled robust automation of the consolidation of the ECG signal data to a feature set with visualization of the temporal orientation of the $P, Q, R, S$, and T waves as illustrated in Figure 1. The feature set for developing the ARFF file was derived from the temporal organization of the following four aspects of the ECG signal:

- $\mathrm{P}$ wave maximum temporal differential relative to the associated $\mathrm{R}$ wave maximum

- $\mathrm{Q}$ wave minimum temporal differential relative to the associated $\mathrm{R}$ wave maximum

- $\mathrm{S}$ wave minimum temporal differential relative to the associated $\mathrm{R}$ wave maximum

- $\mathrm{T}$ wave maximum temporal differential relative to the associated $\mathrm{R}$ wave maximum

A multilayer perceptron neural network was generated by WEKA as illustrated in Figure 2, which consists of four input layer nodes, three hidden layer nodes, and two output layer nodes. The multilayer perceptron neural network achieved a classification accuracy of 95\% for the distinction of two subjects based on their recorded ECG signals. The model required 0.1 seconds to be built. With respect to the derived confusion matrix one instance of the first subject is misclassified as the second subject. Further investigation is conducted with respect to the reduction of attributes. In some scenarios, decreasing the number of attributes has enhanced performance for the machine learning model [19].

Two other machine learning scenarios were addressed with respect to the multilayer perceptron neural network. The first reduced feature set consisted of only the $\mathrm{Q}$ wave minimum temporal differential relative to the associated $\mathrm{R}$ wave maximum and $\mathrm{S}$ wave minimum temporal differential relative to the associated $\mathrm{R}$ wave maximum. The second reduced feature set consisted of $\mathrm{P}$ wave maximum temporal differential relative to the associated $\mathrm{R}$ wave maximum and $\mathrm{T}$ wave maximum temporal differential relative to the associated $\mathrm{R}$ wave maximum.

For the first reduced feature set was comprised of only the $\mathrm{Q}$ wave minimum temporal differential relative to the associated $\mathrm{R}$ wave maximum and $\mathrm{S}$ wave minimum temporal differential relative to the 


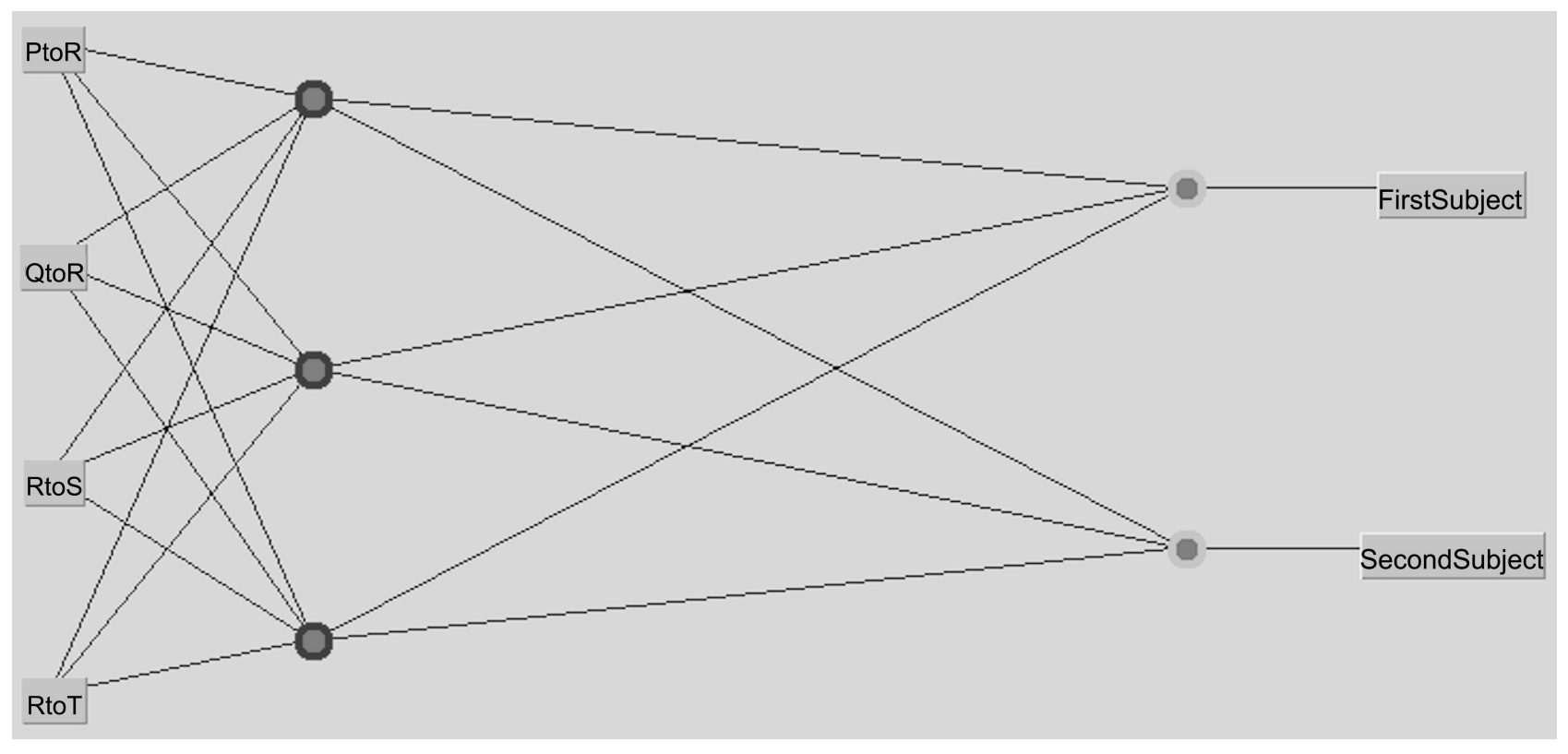

Figure 2. A multilayer perceptron neural network for distinguishing subjects based on their respective ECG signals.

associated $\mathrm{R}$ wave maximum the multilayer perceptron achieved $90 \%$ classification accuracy. The model required 0.06 seconds to develop. With regards to the confusion matrix one instance of the first subject was misclassified as the second subject, and one instance of the second subject was misclassified as the first subject.

The second reduced feature set was comprised of the $\mathrm{P}$ wave maximum temporal differential relative to the associated $\mathrm{R}$ wave maximum and $\mathrm{T}$ wave maximum temporal differential relative to the associated $\mathrm{R}$ wave maximum achieved $95 \%$ classification accuracy through the application of the multilayer perceptron neural network. Notably, this model required 0.01 seconds to be developed. The confusion matrix reveals one instance of the first subject misclassified as the second subject.

These findings warrant the consideration of additional machine learning algorithms. Multiple other machine learning algorithms, such as the J48 decision tree, support vector machine, naïve Bayes, K-nearest neighbors, and logistic regression, are proposed to ascertain the machine learning algorithm that attains the optimal combination of classification accuracy and time to build the model. The comprehensive determination of the optimal combination of classification accuracy and time to build the model provides significant insight with future architectures for further implementation [19, 22].

Another challenge of the acceptance of the amalgamation of biometrics with respect to the application of the ECG signal with the use of machine learning is the issue of trust with respect to the resultant machine learning classification output, such as classification accuracy [23, 24]. Especially for the field of biometrics, there are experts extrinsic to the machine learning skills set, such as cardiologist and even members of the intelligence community. The amalgamation of diverse experts from outside the field of machine learning presents the issue of trust toward the capabilities of the machine learning algorithm achieving distinctive classification accuracy. For these experts a machine learning algorithm can constitute an effective black box $[23,24]$. Further research endeavors can emphasize machine learning algorithms that provide additional visualization techniques.

\section{CONCLUSIONS}

The research objective for the application of the multilayer perceptron neural network to differentiate subjects through the temporal organization of their available ECG signal data has been satisfied. In partic- 
ular, the multilayer perceptron neural network achieved with a reduced feature set emphasizing the temporal differential of the $\mathrm{P}$ wave maximum and $\mathrm{T}$ wave maximum relative to the $\mathrm{R}$ wave maximum provided superior performance in consideration of both classification accuracy and time to develop the model by comparison to the use of all four attributes and the temporal differential of the $\mathrm{Q}$ wave minimum and $\mathrm{S}$ wave minimum relative to the $\mathrm{R}$ wave maximum. The achievement of the research objective provides additional insight with respect to the opportunity of the temporal organization of the ECG signal and robust machine learning algorithms, such as the multilayer perceptron neural network, for biometric applications.

Additionally, the research demonstrates relevant insight for the domain of cardiac health. If a subject's current ECG signal is differentiated relative to a historic ECG signal by machine learning classification beyond a prescribed threshold, more sophisticated cardiology resources may be applied. This perspective may enable advanced intervention prior to the perception of more severe and notable symptoms.

\section{CONFLICTS OF INTEREST}

The authors declare no conflicts of interest regarding the publication of this paper.

\section{REFERENCES}

1. Woodward Jr., J.D. (2001) Biometrics: Facing Up to Terrorism. RAND Arroyo Center, Santa Monica. https://apps.dtic.mil/sti/citations/ADA406181

2. Silva, H., Lourenço, A., Canento, F., Fred, A. and Raposo, N. (2013) ECG Biometrics: Principles and Applications. Proceedings of BIOSIGNALS 6th International Conference on Bio-Inspired Systems and Signal Processing, Barcelona, 11-14 February 2013, 215-220. https://www.scitepress.org/Papers/2013/42432/42432.pdf

3. Israel, S.A. and Irvine, J.M. (2012) Heartbeat Biometrics: A Sensing System Perspective. International Journal of Cognitive Biometrics, 1, 39-65. https://doi.org/10.1504/IJCB.2012.046514

4. Hurst, J.W. (1998) Naming of the Waves in the ECG, with a Brief Account of Their Genesis. Circulation, 98, 1937-1942. https://doi.org/10.1161/01.CIR.98.18.1937

5. Barold, S.S. (2003) Willem Einthoven and the Birth of Clinical Electrocardiography a Hundred Years Ago. Cardiac Electrophysiology Review, 7, 99-104. https://doi.org/10.1023/A:1023667812925

6. Patel, S., Park, H., Bonato, P., Chan, L. and Rodgers, M. (2012) A Review of Wearable Sensors and Systems with Application in Rehabilitation. Journal of Neuroengineering and Rehabilitation, 9, 1-17.

https://doi.org/10.1186/1743-0003-9-21

7. LeMoyne, R. and Mastroianni, T. (2020) Machine Learning Classification for Network Centric Therapy Utilizing the Multilayer Perceptron Neural Network. In: Vang-Mata, R., Ed., Multilayer Perceptrons. Theory and Applications, Nova Science Publishers, Hauppauge, 39-76.

8. LeMoyne, R. and Mastroianni, T. (2020) Waikato Environment for Knowledge Analysis (WEKA) a Perspective Consideration of Multiple Machine Learning Classification Algorithms and Applications. In: LeMoyne, R. and Mastroianni, T., Eds., Applied Software Development with Python \& Machine Learning by Wearable \& Wireless Systems for Movement Disorder Treatment via Deep Brain Stimulation, World Scientific Publishing, Singapore, 137-180. https://doi.org/10.1142/9789811235962_0006

9. Munakata, T. (2008) Fundamentals of the New Artificial Intelligence: Neural, Evolutionary, Fuzzy and More. Springer Science \& Business Media, London.

10. Hall, M., Frank, E., Holmes, G., Pfahringer, B., Reutemann, P. and Witten I.H. (2009) The WEKA Data Mining Software: An Update. ACM SIGKDD Explorations Newsletter, 11, 10-18. https://doi.org/10.1145/1656274.1656278

11. Witten, I.H., Frank, E. and Hall M.A. (2011) Data Mining: Practical Machine Learning Tools and Techniques. 
Morgan Kaufmann, Burlington.

12. WEKA. http://www.cs.waikato.ac.nz/ ml/weka

13. Pinto, J.R., Cardoso, J.S. and Lourenço, A. (2018) Evolution, Current Challenges, and Future Possibilities in ECG Biometrics. IEEE Access, 6, 34746-34776. https://doi.org/10.1109/ACCESS.2018.2849870

14. Wellens, H.J. (1986) The Electrocardiogram 80 Years after Einthoven. Journal of the American College of Cardiology, 7, 484-491. https://doi.org/10.1016/S0735-1097(86)80457-0

15. Zipes, D.P. (2000) Clinical Application of the Electrocardiogram. Journal of the American College of Cardiolo$g y, 36,1746-1748$. https://doi.org/10.1016/S0735-1097(00)00971-2

16. LeMoyne, R. and Mastroianni, T. (2020) Applied Software Development with Python \& Machine Learning by Wearable \& Wireless Systems for Movement Disorder Treatment via Deep Brain Stimulation. World Scientific Publishing, Singapore. https://doi.org/10.1142/12249

17. LeMoyne, R., Kerr, W.T., Zanjani, K. and Mastroianni, T. (2014) Implementation of an iPod Wireless Accelerometer Application Using Machine Learning to Classify Disparity of Hemiplegic and Healthy Patellar Tendon Reflex Pair. Journal of Medical Imaging and Health Informatics, 4, 21-28.

https://doi.org/10.1166/jmihi.2014.1219

18. LeMoyne, R., Kerr, W., Mastroianni, T. and Hessel, A. (2014) Implementation of Machine Learning for Classifying Hemiplegic Gait Disparity through Use of a Force Plate. Proceedings of the 13 th Annual International Conference of the IEEE Machine Learning and Applications (ICMLA), Detroit, 3-6 December 2014, 379-382. https://doi.org/10.1109/ICMLA.2014.67

19. LeMoyne, R. and Mastroianni, T. (2020) Machine Learning Classification of Essential Tremor Using a Reach and Grasp Task with Deep Brain Stimulation System Set to "On" and "Off” Status. In: LeMoyne, R. and Mastroianni, T., Eds., Applied Software Development with Python \& Machine Learning by Wearable \& Wireless Systems for Movement Disorder Treatment via Deep Brain Stimulation, World Scientific Publishing, Singapore, 181-205. https://doi.org/10.1142/9789811235962_0007

20. PhysioNet. https://www.physionet.org/about/\#physionet

21. Goldberger, A.L., Amaral, L.A., Glass, L., Hausdorff, J.M., Ivanov, P.C., Mark, R.G., Mietus, J.E., Moody, G.B., Peng, C.K. and Stanley, H.E. (2000) PhysioBank, PhysioToolkit, and PhysioNet: Components of a New Research Resource for Complex Physiologic Signals. Circulation, 101, e215-220.

https://doi.org/10.1161/01.CIR.101.23.e215

22. LeMoyne, R., Mastroianni, T., Whiting, D. and Tomycz, N. (2019) Assessment of Machine Learning Classification Strategies for the Differentiation of Deep Brain Stimulation “On” and "Off” Status for Parkinson's Disease Using a Smartphone as a Wearable and Wireless Inertial Sensor for Quantified Feedback. In: LeMoyne, R., Mastroianni, T., Whiting, D. and Tomycz, N., Eds., Wearable and Wireless Systems for Healthcare II, Springer, Singapore, 113-126. https://doi.org/10.1007/978-981-13-5808-1_9

23. Ribeiro, M.T., Singh, S. and Guestrin, C. (2016) “Why Should I Trust You?” Explaining the Predictions of Any Classifier. Proceedings of the 22nd ACM SIGKDD International Conference on Knowledge Discovery and Data Mining, San Francisco, 13-17 August 2016, 1135-1144. https://doi.org/10.1145/2939672.2939778

24. Baehrens, D., Schroeter, T., Harmeling, S., Kawanabe, M., Hansen, K. and Müller, K.R. (2010) How to Explain Individual Classification Decisions. Journal of Machine Learning Research, 11, 1803-1831.

https://www.jmlr.org/papers/volume11/baehrens10a/baehrens10a 